\title{
La S.O.T.EST sera à Lons-le-Saunier en 94. Et si on parlait un peu des vins du Jura?
}

Vous connaissez tous les termes de notre credo. On ne parle bien que de ce qu'on connaît parfaitement. Or l'Alsace, notre milieu et la Bourgogne, notre voisine sont pour nous terres de découvertes assez faciles (parce que proches) terres de mission, terres d'investigations, terres familières dont on finit par connaitre en toutes saisons non seulement les reliefs mais aussi l'heure d'hiver au cours de laquelle le moindre et ultime rayon de soleil transforme l'ancienne toile moussue et maussade sous la pluie en en enchantement tombé d'ors verts et de bronze carillonnant.

Les autres régions viticoles ne sont pas ignorées, sûrement pas, mais peut être un peu moins investies parce que plus lointaines, moins à portée d'un saut en voiture. Il ne se passe pourtant pas une année sans que nous évoquions le Bordelais, (à plusieurs reprises), les vins du Rhône (sud et nord), la Provence... Et le Jura?

Là il nous faut confesser notre faute. Le Jura est proche (autant en tous cas que la Bourgogne), riche en découvertes, varié, intéressant, petit certes, mais si dense en richesses que l'ignorer davantage relève de la faute. Faute que nous ne commettrons pas. Quelques mensurations pour commencer :

2000 ha de vignes (c'est petit petit), Schlumberger à Guebwiller cultive 130 ha à lui seul.

50000 à $80000 \mathrm{hl}$ de vin par an. La cave coopérative d'Eguisheim en fait probablement plus. C'est aussi $1 \%$ de la production nationale.

4 appellations contrôlées minuscules: Arbois, Château-Chalon, Étoile, Côtes du Jura.

1 Association Oenologique alsacienne. Adresse : R. Gsell, 4, quai du Maire Dietriech, 67000 Strasbourg
8 vins ou boissons différents.

1 vin de réputation mondiale : le ChâteauChalon.

$1200 \mathrm{~mm}$ de pluie par an en moyenne contre moins de la moitié dans la région de Colmar et Rouffach.

Températures variant de $5^{\circ} 8$ à $15^{\circ}$ (moyennes décembre et juillet)

Voici pour l'essentiel.

Une lecture superficielle et une volonté délibérée d'ignorer le Jura pourraient conduire à la conclusion suivante : ce petit terroir, haut perché, au climat un peu sévère produit des vins dont l'intérêt ne peut être que relatif. Il en est tout autrement.

Le Jura cultive deux cépages blancs d'un très grand intérêt. D’abord le Savagnin, cépage souverain, qui ne s'extériorise nulle part aussi brillamment qu'ici où il donne naissance au Château-Chalon que les plus grands spécialistes de tous les temps classent parmi les 5 meilleurs vins blancs (tranquilles) du monde. Ce n'est pas rien.

Je signale que le Gewürtztraminer est la version "rose améliorée" du Savagnin et que le Klevner de Heiligenstein pourrait être son frère (ce dernier ne saurait lui faire ombrage étant réduit à 15 ha et vinifié autrement). On se perd en hypothèses sur l'origine de ce vin et comme en Alsace, la légende veut qu'il nous vienne de Hongrie dans les bagages d'un comte guerrier, (pas le même qu'en Alsace : Schwendi). Toujours est-il que ce vin blanc qui fait sa malolactique, séjourne 6 ans et 3 mois en fût de chêne. La porosité du bois permet une petite circulation d'air et la formation du fameux "voile" à l'abri duquel le vin prend couleur ef ce goût inimitable qui fait son charme.

Le second cépage n'est autre que le Chardonnay... Extraordinaire Chardonnay qui fait les grands Champagnes, les superbes Meursault, Puligny, Chassagne, Corton Charlemagne, Montrachet... qui prudemment mais sans doute obs- tinément lorgne sur le piémont des Vosges. En effet, ce qui réussit si bien à Chablis, en Côte d'Or, ne peut pas ne pas réussir en Alsace. Toujours est-il que le Chardonnay du Jura, longtemps interdit, parce que jugé médiocre (récoltes trop abondantes) fut réhabilité en 1774. Depuis ... il prospéra tant bien que mal, mais connaît aujourd'hui un regain de ferveur parce qu'il est sec et fruité... mais surtout parce qu'il ne dédaigne point de convoler en justes noces avec un brin de Savagnin.

Lorsque le fût de Chardonnay se vide régulièrement un peu, par le seul phénomène de l'évaporation, on le remplit systématiquement autant que régulièrement avec du Savagnin. C'est superbe... Nous voici par conséquent cousins par les cépages de nos voisins du Jura et si on ajoute le Pinot Noir à la gamme, on peut dire que seuls le Poulsard (rouge) et le Trousseau (rouge) sont inconnus dans le Grand Est (Bourgogne, Champagne, Alsace). L'Art et le Vin ${ }^{1}$ se propose de faire une incursion gustative du côté de ces grands vins et de vous convier en avril ou mai à un stage où nous déclinerons l'ensemble des vertus de ce beau terroir. Pour les amateurs de bonnes lectures, nous ne saurions que recommander le livre de Jean-Pierre Pidoux "Le vignoble et les grands vins du Jura". Son préfacier, Pierre Brantus, écrit à propos du Savagnin : "il n'est à ma connaissance que très peu de vins dans le monde susceptibles de vieillir convenablement pendant plusieurs siècles.

Incomparable Savagnin ! Lui, a ce privilège, privilège jurassien.

"Connais-tu ce nectar fameux Que le Jura tient en ses treilles Et qui, tombant de la bouteille Se boit d'abord avec les yeux?" 